\title{
Propuesta de un Modelo de Humanización basado en las Competencias Personales: Modelo HUMAS
}

\author{
María del Carmen Pérez-Fuentes ${ }^{1}$, María del Mar Molero Jurado ${ }^{1}$, \\ Iván Herrera Peco ${ }^{2}$, Nieves Fátima Oropesa Ruiz ${ }^{1}$, y \\ José Jesús Gázquez Linares ${ }^{3}$ \\ ${ }^{1}$ Universidad de Almería (España); ${ }^{2}$ Universidad Alfonso X el Sabio (España); \\ ${ }^{3}$ Universidad Autónoma de Chile
}

\begin{abstract}
A pesar de que hoy en día el concepto de Humanización está muy presente en el entorno de los cuidados, no existe una definición clara sobre qué puede entenderse por humanización de los cuidados. Con el objetivo de colaborar en la concreción de un término que posee una clara composición multifactorial, se plantea la creación de un modelo que permita explicar las competencias y actitudes que debe desarrollar un profesional sanitario para incorporar la Humanización en el ejercicio de su profesión. En el modelo propuesto, la Humanización se define como un conjunto de competencias personales que permiten desarrollar la actividad profesional, dentro del ámbito sanitario, velando por la dignidad y el respeto hacia el ser humano. Es, por tanto, una actividad enfocada a la mejora del cuidado de la salud, tanto física como mental y emocional, dirigida tanto a pacientes y familiares, como a los propios profesionales sanitarios. El modelo teórico propuesto HUMAS, está centrado en los procesos psicológicos, en las competencias personales que capacitan a los profesionales sanitarios para ofrecer al paciente una atención de calidad. Así, el profesional, contando con todos los recursos disponibles en el sistema sociosanitario y en el entorno del paciente, además requiere de la adquisición y el desarrollo de competencias personales entre las que se encuentra la disposición al optimismo, la sociabilidad, la compresión emocional, la autoeficacia y la afectación, entendidas como esenciales para contribuir a un desempeño profesional positivo, basado en la humanización, como es el modelo HUMAS.
\end{abstract}

Palabras clave: competencias personales; humanización de los cuidados; profesionales sociosanitarios; salud ocupacional; modelo HUMAS.

Proposal for a Humanization Model based on Personal Competencies: HUMAS Model. Although today the concept of humanization is very present in the care environment, there is no clear definition of what can be understood by humanizing care. The aim of collaborating in the realization of a term that has a clear multifactorial composition, the creation of a model to explain the skills and attitudes that must be developed by a healthcare professional to incorporate Humanization in the exercise of his profession. In the proposed model, humanization is defined as a set of personal competences that allow the development of professional activity, within the health field, ensuring dignity and respect for the human being. It is therefore an activity focused on the improvement of health care, both physical, mental and emotional, aimed at patients and relatives, as well as the healthcare professionals themselves. The theoretical model proposed HUMAS focuses on psychological processes, on the personal skills that train health professionals to offer the patient quality care. Thus, the professional, with all the resources available in the social and health system and in the patient's environment, it also requires the acquisition and development of personal skills, including optimism, sociability, emotional understanding, self-efficacy and involvement, understood as essential to contribute to positive professional performance, based on humanization, such as the HUMAS model.

Keywords: personal skills; humanization of care; socio-health professionals; occupational health; HUMAS model.

Correspondencia: María del Carmen Pérez Fuentes. Departamento de Psicología Evolutiva y de la Educación. Universidad de Almería. Ctra. La Cañada de San Urbano s/n, C.P. 04120. Almería (España). E-mail: perezfuentes@ual.es 
A pesar de que hoy en día el concepto de la Humanización está muy presente en el entorno de los cuidados, e incluso ha sido rápidamente acogido entre los profesionales de la salud, no existe una definición clara sobre la Humanización de los cuidados. Esta falta de operacionalización e incluso de concreción del concepto, hace que éste tenga muchas barreras que dificultan su desarrollo. Definir algo, máxime atendiendo a la vaguedad del término, siempre resulta complejo, sin embargo, es posible valorar el entorno de la Humanización teniendo en cuenta a los agentes implicados y a las acciones que se vienen desarrollando.

Con respecto a los agentes implicados, la Humanización de los cuidados se fundamenta en la interacción entre varios actores: (1) pacientes y familiares,

(2) profesionales sanitarios y cuidadores no profesionales, y (3) gestores. Si bien la interacción entre todos ellos es esencial para que se pueda dar un entorno de trabajo y del cuidado que cumpla con los objetivos y se adecue a la misión de un sistema sanitario, consideramos que es de vital importancia, para la Humanización del cuidado, la participación activa de los profesionales sanitarios, puesto que son los encargados de ofrecer ese cuidado a los pacientes.

En relación a las acciones que se puedan llevar a cabo en el contexto de la humanización del cuidado, dichas actuaciones suelen enfocarse en tres direcciones: de una parte, hacia el liderazgo de las organizaciones sanitarias; de otro, hacia las competencias relacionales, humanísticas y éticas y, por último, hacia la organización humanizada, entendida como aquella que debe cuidar su estilo, identidad e incluso valores, como puede apreciarse, por mencionar algunas cuestiones referidas a la estructura de los centros sanitarios, el hecho de la propia arquitectura, la decoración o señalítica, sin olvidar que los profesionales presenten una identificación exclusiva y representativa del ámbito sanitario (Kotzer, Zacharakis, Raynolds, y Buenning, 2011; Roohafza et al., 2009).

En el momento actual, parece que la definición más ampliamente extendida con respecto a la Humanización se asocia directamente con la calidad percibida de un servicio asistencial prestado: número de actividades realizadas, protocolos desarrollados con éxito, medidas aplicadas, etc. Sin embargo, entendemos que la humanización del cuidado, entendido como un estilo en la relación entre personas, donde intervienen diferentes procesos psicológicos, no puede entenderse únicamente de un modo tan aséptico, ya que desde esta perspectiva parecen obviarse las competencias personales del propio profesional, poniéndose el acento, para medir la Humanización, tan sólo en los productos de las acciones desarrolladas por dichos profesionales. En base a lo anterior, proponemos una definición de la Humanización del cuidado más enfocada al conjunto de competencias personales que permiten desarrollar la actividad profesional, dentro del ámbito sanitario, respetando y velando por la dignidad y el respeto al ser humano (PérezFuentes et al., 2019b). Es, por tanto, una actividad orientada a la mejora del cuidado 
de la salud, tanto física como mental y emocional, dirigida tanto hacia los pacientes como a los propios profesionales sanitarios.

\section{Panorama actual de la Humanización en salud}

En el momento actual, la humanización de los cuidados se encuentra desarrollándose a través de diferentes planes e iniciativas, algunas de las cuales se dirigen a una serie de intervenciones sobre el ambiente: modificación de luces o eliminación de ruidos (Kotzer et al., 2011), acciones enfocadas a la decoración de las paredes en pediatría (Monti et al., 2012), sin olvidar la incorporación de elementos de entretenimientos en las salas de espera, como pantallas táctiles con juegos interactivos para amenizar la espera en el área pediátrica (Biddiss, McPherson, Shea, y McKeever, 2013). Además, en cuanto a actuaciones se dan numerosas experiencias en las cuales se introducen animales como parte de la terapia, como se puede apreciar en diversos ejemplos de la literatura científica, tales como los descritos por Branson, Boss, Padhye, Trötscher, y Ward (2017), Gertz y Rabinowitz (2017), Mani y Weese (2016), y Moretti et al. (2010), entre otros.

Otras de las intervenciones están enfocadas a la incorporación de acciones derivadas de profesionales, pudiendo ser no sanitarios, que ofrecen a los pacientes un momento de entretenimiento e incluso de relax, dentro del entorno sanitario, como las actuaciones de payasos, que ofrecen una interesante perspectiva a la hora de reducir el estrés en el entorno pediátrico (Barkmann, Siem, Wessolowski, y SchulteMarkwort, 2013), y de utilidad también para otro tipo de pacientes (no sólo para los niños) (Boscarelli, 2017; Dionigi y Canestrari, 2016). Otro enfoque que se da habitualmente dentro de las acciones de la Humanización es el de la musicoterapia, donde la música resulta efectiva por ejemplo a la hora de generar motivación y proporcionar confort al paciente oncológico, tras recibir el tratamiento (da Silva, Marcon, y Sales, 2014).

Además, no pueden olvidarse las acciones dirigidas al personal sanitario, desde cambios en el uniforme, como por ejemplo sucede en áreas pediátricas al cambiar el color de la ropa que se viste (Roohafza et al., 2009), hasta las intervenciones centradas en los profesionales sanitarios, donde se ofrece formación, ya sea a modo de talleres o seminarios, dirigidas principalmente a la mejora de las habilidades de comunicación (Darban, Balouchi, Narouipour, Safarzaei, y Shahdadi, 2016; Tripodi et al., 2019).

En resumen, y a pesar de que se están desarrollando numerosas acciones encaminadas a desarrollar la Humanización del cuidado, es importante destacar que muy pocas de ellas tienen como objetivo principal el hecho de proporcionar formación e instrucción en la mejora de las competencias personales, que pueden ser relacionadas con el propio concepto de la prestación del cuidado por parte de los profesionales sanitarios, 
sino que más bien están orientadas a la introducción de modificaciones sobre el entorno o ambiente que existe dentro del propio sistema sanitario, y no sólo en los centros sanitarios.

\section{El profesional sanitario y las actitudes básicas en la humanización}

Dentro de un proceso asistencial, y asociado a los profesionales sanitarios, se puede considerar que la Humanización del cuidado debería estar integrada por diversos aspectos, entre los que mencionamos los siguientes: personalización de la atención; calidad en el trato; buena comunicación, aportando información clara y eficaz; transmitir seguridad y confianza; proporcionar una atención integral y holística; demostrar dignidad y respeto por los derechos de las personas; comprender las necesidades y expectativas de los pacientes (Busch, Moretti, Travaini, Wu, y Rimondini, 2019; Greene, Tuzzio, y Cherkin, 2012; Luiz, Caregnato, y Costa, 2017; Medeiros et al., 2016; Reeve, Lynch, Lloyd-Williams, y Payne, 2012; Shin y Kang, 2019; Tripodi et al., 2019).

Atendiendo a lo anterior, parece claro que la Humanización de los cuidados implica que el personal sanitario presente un conjunto de recursos tanto sociales, como personales y emocionales que defina su práctica profesional, facilitando el desempeño laboral y permitiendo que la persona, el paciente, adopte un papel activo en el proceso de recuperación. Otros autores vienen señalando, desde hace tiempo, en el mismo sentido, que desde el sistema sanitario se deben ofrecer herramientas al paciente para afrontar de manera activa su salud y bienestar (Gerteis, Edgman-Levitan, Daley, y Delbanco, 1993; Gerteis et al., 1993).

Se trata de un enfoque de construcción conjunta de la salud, donde el profesional es capaz de respetar a la persona y de ser sensible a sus necesidades, pero también de proporcionarle herramientas para mejorar la salud desde un enfoque holístico e integral en la atención, basada en la intersubjetividad y la reciprocidad. Es, por tanto, el conjunto de competencias que permitirán que el paciente pueda ser considerado realmente como el centro del sistema, donde pueda participar activamente del proceso del cuidado de su propia salud, convirtiéndose así en parte activa de su proceso, junto a los profesionales sanitarios (Pérez-Fuentes et al., 2019b).

\section{¿Podemos evaluar la humanización?}

Actualmente, la medición de la Humanización del cuidado, entendida como una dimensión inherente al profesional sanitario que se sustenta en sus competencias personales y no técnicas, no se lleva a cabo en el ámbito de la salud. Más bien la Humanización, hasta el momento ha sido entendida como aquella acción o cualidad centrada en los resultados obtenidos de la aplicación de los planes institucionales de humanización. Al medirla podemos encontrar que la valoración que se desarrolla posee una clara inspiración procedente de la evaluación de los procesos de calidad derivados de las normas ISO (Organización Internacional de Normalización) o del modelo EFQM 
(Modelo Europeo de Excelencia Empresarial), donde se valoran, por un lado, el nivel de ejecución de las actividades programadas y, por otro, el grado de cumplimiento de cada plan de acción definido (EFQM, 2015; ISO/IEC, 2019). Los grados de cumplimiento se medirán, a su vez, a partir de los porcentajes de las actividades que hayan sido ejecutadas, puestas en marcha, reprogramadas o, incluso, suprimidas.

Por otro lado, y en este mismo sentido, encontramos propuestas como las planteadas por el proyecto HUCI (Humanización de los Cuidados Intensivos), donde en su "Manual de Buenas Prácticas" recoge una serie de 159 medidas a implementar en las Unidades de Cuidados Intensivos, para valorar si se cumplen unos estándares de humanización en dichas Unidades (Velasco, Heras, Ortega, y Gómez, 2017). La forma de medir o valorar estas buenas prácticas de humanización es realizando un Checklist y, en gran medida, se fundamenta en la realización o no de determinados procesos o acciones, más que en las competencias personales de los profesionales sanitarios que aportan los cuidados al paciente.

Por tanto, a la hora de valorar posibles formas de evaluación de la Humanización del cuidado a través de las competencias personales de los profesionales sanitarios implicados en el proceso de cuidar, debemos indicar que no existen y resulta una necesidad el valorar y analizar las competencias personales, no técnicas, de los principales agentes que desarrollan el Cuidado del paciente, los profesionales sanitarios.

\section{Modelo de humanización en el profesional sanitario: el Modelo HUMAS}

Con el objetivo de colaborar en la concreción de un término que posee una clara composición multifactorial, se plantea la creación de un modelo que permite explicar la Humanización del cuidado ofrecido por los profesionales sanitarios.

Es importante destacar que los aspectos que definen la Humanización en este modelo, no se asocian con el conocimiento técnico que, de todos es conocido, es algo que poseen en un elevado grado todos los profesionales sanitarios que desarrollan su actividad en el Sistema Sanitario, puesto que la formación recibida se encamina a proporcionar un alto grado de conocimiento en los procesos y uso de la tecnología. Este modelo se enfoca a aspectos como son la afectación, la autoeficacia, la comprensión emocional, la disposición al optimismo y la sociabilidad (Figura 1).

En el modelo propuesto, la Humanización se define como un conjunto de competencias personales que permiten desarrollar la actividad profesional, dentro del ámbito sanitario, respetando y velando por la dignidad y el respeto al ser humano. Es, por tanto, una actividad enfocada a la mejora del cuidado de la salud, tanto física como mental y emocional, tanto hacía los pacientes como a los propios profesionales sanitarios. 
Figura 1. Modelo de Humanización en el profesional sanitario (Modelo HUMAS)

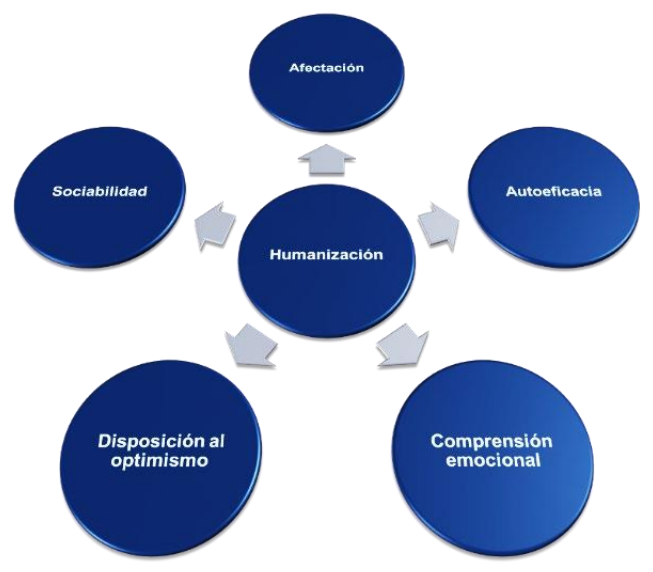

¿Qué se entiende por optimismo?

El optimismo es la creencia de un individuo sobre la probabilidad de obtener resultados positivos en el futuro, la tendencia de un individuo a creer en los mejores resultados posibles ante la incertidumbre (Bar-On, 2006; Seligman, 2006). Por tanto, se podría decir que el optimismo es el pilar del pensamiento positivo.

El optimismo es un estilo de pensamiento, no una parte permanente del individuo, por tanto, es posible trabajar para el cambio. Además, también es el resultado de diferentes procesos de interacción social y experiencias. Así, combinando las perspectivas psicológica y social, sería posible un abordaje completo y equilibrado del optimismo.

Salvando la famosa frase del vaso medio lleno o medio vacío, Oscar Wilde dijo una vez que la diferencia entre un optimista y un pesimista es que el optimista ve la rosquilla, mientras que el pesimista observa el agujero. Es obvio que el pesimismo tiene su lugar y, en ocasiones, se puede suponer un recurso adaptativo, pero demasiada negatividad puede socavar el trabajo, las amistades y la salud. De hecho, detenerse en lo negativo aumenta el estrés (Segerstrom, Carver, y Scheier, 2017).

La creciente importancia de incorporar el positivismo en el lugar de trabajo es reconocida por los investigadores (Bakker, Rodríguez-Muñoz, y Derks, 2012). Existe evidencia que vincula el concepto con la mejora en el desempeño de los trabajadores. ¿Con quién preferirías trabajar, con alguien que te haga la jornada más fácil, o cerca de alguien que pasa el día quejándose por todo?

Hay una gran diferencia en estos dos puntos de vista, fundamentalmente sobre el impacto en el trabajo, la salud y la actitud hacia la vida, en general. Las investigaciones demuestran que el optimismo puede prevenir la depresión, aumentar la conexión social, 
aumentar el rendimiento en el trabajo, atraer el éxito y hacer que sea más resistente ante los contratiempos (Carver y Scheier, 2014; Segerstrom et al., 2017; Seligman, Schulman, y Tryon, 2007). La negatividad restringe el pensamiento, nos pone a la defensiva y nos impide ver el panorama general (Wersebe, Lieb, Meyer, Hofer, y Gloster, 2018). No es de extrañar entonces que un enfoque más positivo genere resultados más positivos. Energiza, amplía las oportunidades, descubre soluciones, mejora en gran medida el equilibrio entre la vida laboral y personal. Por otra parte, los profesionales de la salud, en continua interacción con compañeros, pacientes y familiares, y a menudo con un desempeño multitarea con plazos reducidos de tiempo son más vulnerables al agotamiento (Dall'Ora, Griffiths, Ball, Simon, y Aiken, 2015; Martos et al., 2018; Molero, Pérez-Fuentes, Gázquez, Simón, y Martos, 2018b). En lo referente a la prevención de los síntomas del agotamiento, el optimismo y sus efectos amortiguadores tienen mucho que aportar (Vizoso y Arias, 2018).

\section{¿Qué se entiende por sociabilidad?}

La interacción social, aunque está eminentemente presente en todas las situaciones cotidianas del ser humano, es en las profesiones sanitarias donde las relaciones inevitablemente se intensifican (Lown, McIntosh, Gaines, McGuinn, y Hatem, 2016). Así, los rasgos de sociabilidad son características que poseen las personas que fomentan las relaciones efectivas (Bar-On, 2006). Podemos diferenciar dos tipos de perfiles: aquellos que naturalmente muestran rasgos de sociabilidad, como una actitud amigable y abierta, autocontrol, adaptabilidad, integridad y espíritu de equipo, y aquellos que adoptan habilidades sociales con el tiempo. Los primeros suelen presentarse como líderes motivados, que son una pieza clave para el desempeño de su labor profesional, pero también un activo en su desarrollo personal y bienestar, consigo mismo y con los demás (Seligman, 2006).

En una profesión en la que se debe velar por el bienestar de otros, parece que las cualidades altruistas son necesarias, pero deben aprender a convivir de forma equilibrada con las demandas propias del rigor en la atención y el mantenimiento de lo que se define como un "trato profesional". En ocasiones, la tendencia hacia la conservación del rol profesional y el esfuerzo por protegerse de cualquier atisbo de

vulnerabilidad en la relación con el otro, los profesionales de la salud pueden llegar a utilizar escudos que los protejan de actitudes y comportamientos humanistas y prosociales.

Así, suele ocurrir que la promoción de la conducta prosocial y el altruismo en el contexto de la salud es contradictoria. Al respecto, se recomienda un enfoque de atención clínica que sea prosocial y empático. Diferentes estudios muestran que el entrenamiento en atención plena, auto-reflexión y habilidades sociales puede ayudar a los profesionales de la salud a reconocer, regular y demostrar empatía dentro de los encuentros 
clínicos y profesionales (Asuero, Blanco, Pujol-Ribera, Berenguera, y Queraltó, 2013; Oro, Esquerda, Viñas-Salas, Soler-González, y Pifarré, 2015).

Atendiendo a las consecuencias derivadas, podría decirse que existe una estrecha relación entre las habilidades sociales de los profesionales de la salud y su desempeño laboral (Molero, Pérez-Fuentes, Gázquez, y Barragán, 2018a). Después de todo, independientemente de la competencia técnica de los profesionales sanitarios, la capacidad para tratar con pacientes o familiares e influir en su comportamiento, dependerá más de sus actitudes y habilidades en trato. La promoción del interés propio hacia otra persona en riesgo, es el núcleo de la práctica sanitaria.

\section{¿Qué se entiende por comprensión emocional?}

La comprensión emocional es un aspecto importante del desarrollo emocional. Si las emociones proporcionan información relevante para el desempeño personal y social, entonces las personas con mayor capacidad para identificar, comprender y manifestar sus emociones, se posicionan ventajosamente en la resolución de problemas, sobre todo de comunicación o, simplemente, en la interacción con los demás.

Aunque los profesionales experimentan y muestran emociones en el trabajo, lo cierto es que cada vez con más frecuencia, el lugar de trabajo se presenta como un entorno de tareas automatizadas, inhumano. Los niveles de estrés están en su punto más alto, la digitalización ha acelerado el ritmo de vida a una velocidad casi impersonal. Parece que, ante este panorama, no es humanamente posible mantener la prevalencia de las emociones, con las presiones y los plazos.

Hoy en día, nos enfrentamos al desafío de "Re-Humanizar" el lugar de trabajo. Este proceso comienza con la adquisición de un nuevo tipo de inteligencia emocional. Para ello, sería necesario desarrollar un nuevo vocabulario emocional, es decir, ser capaz no solo de identificar lo que otros están experimentando, sino capaz de comprenderlo. Esto supone el inicio de un cambio positivo en la organización, los grupos, las relaciones interpersonales y el contexto de atención en salud.

Nuestra capacidad para analizar (en lugar de interpretar subjetivamente) las situaciones de manera objetiva y, por lo tanto, para entendernos a nosotros mismos y a otras personas, depende de mantener un equilibrio sano entre lo que pensamos y lo que sentimos, y desde luego, en última instancia, lo que finalmente hacemos. Ser personas emocionalmente inteligentes no es otra cosa que mostrarnos capaces de experimentar, comprender y gestionar eficazmente las emociones (Decety y Fotopoulou, 2015; Howick, Steinkopf, Ulyte, Roberts, y Meissner, 2017; Pérez-Fuentes, Molero, y Gázquez, 2019a).

La inteligencia emocional requiere de un aprendizaje constante, cotidiano, sobre las respuestas emocionales, tanto las propias como las de otros, aprendiendo a reconocer y comprender los sentimientos, y que seamos capaces de ajustar tales respuestas 
a las situaciones y a los agentes de interacción, en nuestra vida diaria, trabajo y relaciones. Otro aspecto interesante de la inteligencia emocional es la precisión a la hora de verbalizar los sentimientos. La inteligencia emocional puede ser incluso más importante que el IQ (Cociente Intelectual) en la capacidad del logro exitoso y el bienestar (Bar-On, 2006; Goleman, 1995). Las personas que han desarrollado una alfabetización emocional (Bisquerra y Pérez, 2007), tendrán más confianza en sí mismos, y mayor comprensión de los demás y, por tanto, empatía.

\section{¿Qué se entiende por autoeficacia?}

Aprender a identificar, comprender y expresar las emociones de manera saludable, incluye la capacidad de concretar el alcance de las propias emociones en la generación de pensamientos y comportamientos, así como la habilidad para inhibir o, en el mejor de los casos, retrasar, las respuestas poco reflexivas ante emociones intensas. Una vez se desarrollan estas habilidades, se adquiere confianza en uno mismo, sabiendo que "tengo lo que necesito" para afrontar con éxito las situaciones difíciles. Estas habilidades emocionales se conocen como autoeficacia emocional y aquellos que carecen de ella pueden tener dificultades para interactuar positivamente con otros (Bar-On, 2006). Además, la falta de autoeficacia emocional puede interferir con su capacidad para lograr objetivos importantes en la vida, como mantener un desempeño adecuado en el ámbito laboral (Extremera, Mérida-López, Sánchez-Álvarez, y Quintana-Orts., 2018; Miao, Humphrey, y Qian, 2016).

La autoeficacia emocional está marcada por la capacidad de manejar las emociones internamente. Es por ello, que una de las características asociadas a la autoeficacia emocional es la expresión de emociones de una manera que sea socialmente aceptable y apropiada a cada situación (Dvash y Shamay-Tsoory, 2014). Finalmente, la autoeficacia emocional implica la capacidad para comprender que las emociones no reflejan con certeza los hechos objetivos. Es decir, entender que los sentimientos son fruto de nuestra experiencia personal, es una fracción subjetiva de la realidad, resultado de la interpretación subjetiva de los acontecimientos.

Resumiendo, ser autoeficaz, emocionalmente hablando, significa sentirse capaz emocionalmente, es decir, que uno se percibe como una persona hábil en la interacción con otros, concretamente para identificar emociones (p. e. detectar emociones negativas o cambios de humor) y, en base a esto, afrontar situaciones específicas que requieren de una respuesta ajustada a la persona y al contexto. 


\section{¿Qué se entiende por afectación?}

Los trabajadores de la salud están sujetos a la experiencia diaria de situaciones extremas que pueden causar estrés en el desempeño de su labor de atención a los pacientes, sobre todo, en casos críticos o de alta complejidad, sea por la gravedad de los síntomas o por las características del paciente (García-Rodríguez, Gutiérrez-Bedmar, BellónSaameño, Juñoz-Bravo, y Fernández-Crehuet, 2015). La enfermería, la medicina (y otras disciplinas afines), figuran entre las profesiones más estresantes, por la responsabilidad que ostentan al manejar el bienestar, y en ocasiones la vida de los pacientes y la proximidad con éstos y sus familiares (Batool, Nishat, y Yaqoob, 2012; López-García, Ruiz-Hernández, Llor-Zaragoza, Llor-Zaragoza, y Jiménez-Barbero, 2018). Exige una gran dedicación en el desarrollo de sus actividades, con mayor probabilidad de padecer problemas de salud y el desarrollo de episodios de estrés, que pueden llegar a cronificarse, en el peor de los casos (Molero et al., 2018a).

La atención en salud supone a veces un entorno desafiante para aquellos que luchan por cumplir obligaciones morales con pacientes, familiares y la comunidad en general. Tales restricciones, pudiendo ser reales o percibidas, dificultan el cumplimiento que los profesionales de la salud tenían previsto con respecto a su responsabilidad moral, o al menos en la forma en que consideran que debería hacerse. En este punto, pueden experimentar ansiedad y sentimiento de culpa que, a menos que se resuelva, puede extenderse a otras facetas de su desarrollo e incluso cronificarse, derivando en trastornos del estado de ánimo, por ejemplo.

Cuando hacemos referencia al término "Afectación" lo primero que nos viene a la cabeza son palabras relacionadas con el afecto. Lo cierto es que, aunque no expresamente, no vamos desencaminados. En ocasiones, un pobre reconocimiento de nuestras emociones se traduce en una gestión y/o regulación limitada de las mismas, hasta el punto de padecer los efectos negativos de nuestra propia vulnerabilidad. Por ejemplo, en situaciones de atención sanitaria, se habla de la importancia de la empatía en la relación con el paciente. Evidentemente, como se indicaba anteriormente, el sentimiento de responsabilidad es una constante dentro de la labor de atención en salud. Sin embargo, un sentido de responsabilidad "poco realista" o poco ajustado a las circunstancias, puede desembocar en una cadena de "afectos" negativos que van desde el nerviosismo, pasando por la ansiedad, el miedo, incluso la culpa (Pérez-Fuentes, Molero, Gázquez, y Oropesa, 2018).

En este sentido, cobra especial relevancia la capacidad de autorregulación emocional en el personal sanitario. Esta es la herramienta que va a calibrar (ajustar) el grado en que nos afecta el hecho de empatizar con los otros, y la respuesta emocional que percibimos (a nivel personal), así como nuestro repertorio conductual (a nivel social). En ambos casos, un bajo control de la Afectación implica consecuentemente un detrimento 
de la calidad de la atención en salud, además de los efectos perjudiciales en el bienestar del profesional.

\section{CONCLUSIONES}

Con el modelo de Humanización propuesto se pretende contribuir al desarrollo y puesta en práctica de la Humanización de los cuidados dentro del contexto sanitario, mejorando la atención a las personas, contando con profesionales sanitarios comprometidos y satisfechos con su desempeño profesional, circunstancia que se verá facilitada mediante la adquisición y desarrollo de las competencias personales necesarias para contribuir a la mejora de la calidad de los cuidados asistenciales en el contexto de la salud.

Este modelo pone el énfasis en el proceso, en la adquisición de competencias personales que permitan la consecución de los resultados vinculados al cuidado humanizado y rescata el papel activo de la persona, tanto del paciente como de los profesionales sanitarios, así como de otras personas implicadas en el proceso de cambio de la salud (como familiares y personas significativas en la vida del paciente), partiendo de las necesidades de los pacientes y contribuyendo a su desarrollo integral desde un enfoque holístico.

Con esta finalidad, se plantea un modelo que está centrado en los procesos psicológicos, en las competencias personales que capacitan a los profesionales sanitarios para movilizar en el paciente la autogestión de sus cuidados, contando con todos los recursos disponibles en el sistema sociosanitario y en el entorno del paciente, pero que requiere de la adquisición, capacitación y desarrollo de competencias personales, que se muestran en el presente modelo como significativas, entre las que se encuentran la disposición al optimismo, la sociabilidad, la compresión emocional, la autoeficacia y la afectación, entendidas como esenciales para contribuir a un desempeño profesional positivo.

\section{REFERENCIAS}

Asuero, A.M., Blanco, T.R., Pujol-Ribera, E., Berenguera, A., y Queraltó, J.M. (2013). Evaluación de la efectividad de un programa de mindfulness en profesionales de atención primaria. Gaceta Sanitaria, 27(6), 521-528. DOI: 10.1016/j.gaceta.2013.04.007

Bakker, A.B., Rodríguez-Muñoz, A., y Derks, D. (2012). La emergencia de la psicología de la salud ocupacional positiva. Psicothema, 24(1), 66-72.

Barkmann, C., Siem, A.K., Wessolowski, N., y Schulte-Markwort, M. (2013). Clowning as a supportive measure in paediatrics-a survey of clowns, parents and nursing staff. BMC Pediatrics, 13(1), 166.

Bar-On, R. (2006). The Bar-On model of emotional-social intelligence (ESI). Psicothema, 18, 1325. 
Batool, I., Nishat, N., y Yaqoob, A. (2012). A work related stress among nurses of public hospitals of AJyK"-Cross-Sectional Descriptive Study. HealthMed, 6(5), 1651-1660.

Biddiss, E., McPherson, A., Shea, G., y McKeever, P. (2013). The design and testing of interactive hospital spaces to meet the needs of waiting children. HERD: Health Environments Research y Design Journal, 6(3), 49-68. DOI: 10.1177/193758671300600305

Bisquerra, R., y Pérez, N. (2007). Las competencias emocionales. Educación XXI, 10, 61-82. Boscarelli, A. (2017). Clown therapy: not only a pediatric matter. Translational Pediatrics, 6(2), 111-112. DOI: $10.21037 /$ tp.2017.03.03

Branson, S.M., Boss, L., Padhye, N.S., Trötscher, T., y Ward, A. (2017). Effects of animal-assisted activities on biobehavioral stress responses in hospitalized children: A randomized controlled study. Journal of Pediatric Nursing, 36, 84-91. DOI: 10.1016/j.pedn. 2017.05.006

Busch, I.M., Moretti, F., Travaini, G., Wu, A. W., y Rimondini, M. (2019). Humanization of Care: Key Elements Identified by Patients, Caregivers, and Healthcare Providers. A Systematic Review. The Patient-Patient-Centered Outcomes Research, 12(5), 1-14. DOI: 10.1007/ s40271-019-00370-1

Carver, C.S., y Scheier, M.F. (2014). Dispositional optimism. Trends in Cognitive Sciences, 18(6), 293-299. DOI: 10.1016/j.tics.2014.02.003

Da Silva, V.A., Marcon, S.S., y Sales, C.A. (2014). Percepções de familiares de pessoas portadoras de câncer sobre encontros musicais durante o tratamento antineoplásico. Revista Brasileira de Enfermagem, 67(3), 408-414. DOI 10.5935/0034-7167.20140054

Dall'Ora, C., Griffiths, P., Ball, J., Simon, M., y Aiken, L.H. (2015). Association of 12 h shifts and nurses' job satisfaction, burnout and intention to leave: findings from a cross-sectional study of 12 European countries. BMJ Open, 5(9), e008331. DOI: 10.1136/bmjopen-2015008331

Darban, F., Balouchi, A., Narouipour, A., Safarzaei, E., y Shahdadi, H. (2016). Effect of communication skills training on the burnout of nurses: a cross-sectional study. Journal of Clinical and Diagnostic Research: JCDR, 10(4), IC01. DOI: 10.7860/JCDR /2016/19312.7667

Decety, J., y Fotopoulou, A. (2015). Why empathy has a beneficial impact on others in medicine: unifying theories. Frontiers in Behavioral Neuroscience, 8, 457. DOI: 10.3389/fnbeh.2014.00457

Dionigi, A., y Canestrari, C. (2016). Clowning in health care settings: The point of view of adults. Europe's Journal of Psychology, 12(3), 473. DOI: 10.5964/ejop.v12i3.1107

Dvash, J., y Shamay-Tsoory, S.G. (2014). Theory of mind and empathy as multidimensional constructs: Neurological foundations. Topics in Language Disorders, 34(4), 282-295. DOI: 10.1097/TLD.0000000000000040

European Foundation for Quality Management (2015). An overview of the EFQM excellence model. Recuperado de: https://www.bqf.org.uk/wp-content/uploads/2015/11/Overview-of-theEFQM-Excellence-Model.pdf

Extremera, N., Mérida-López, S., Sánchez-Álvarez, N., y Quintana-Orts, C. (2018). How Does Emotional Intelligence Make One Feel Better at Work? The Mediational Role of Work Engagement. International Journal of Environmental Research and Public Health, 15(9), 1909. DOI: 10.3390/ijerph15091909

García-Rodríguez, A., Gutiérrez-Bedmar, M., Bellón-Saameño, J.Á., Muñoz-Bravo, C., y Fernández-Crehuet, J.N. (2015). Psychosocial stress environment and health workers in public health: Differences between primary and hospital care. Atención Primaria, 47(6), 359-366. DOI: 10.1016/j.aprim.2014.09.003

Gerteis, M., Edgman-Levitan, S., Daley, J., y Delbanco, T.L. (1993). Understanding and promoting 
patient-centered care. San Francisco, CA: Jossey-Bass.

Gerteis, M., Edgman-Levitan, S., Walker, J.D., Stoke, D.M., Cleary, P.D., y Delbanco, T.L. (1993). What patients really want. Health Management Quarterly: $H M Q$, 15(3), 2-6.

Gertz, A., y Rabinowitz, P.M. (2017). Pet Therapy: Helping Patients Cope. American Family Physician, 96(7), 464-464.

Goleman, D. (1995). Emotional intelligence. New York: Bantam.

Greene, S.M., Tuzzio, L., y Cherkin, D. (2012). A framework for making patient-centered care front and center. The Permanente Journal, 16(3), 49-53.

Howick, J., Steinkopf, L., Ulyte, A., Roberts, N., y Meissner, K. (2017). How empathic is your healthcare practitioner? A systematic review and meta-analysis of patient surveys. BMC Medical Education, 17(1), 136. DOI: 10.1186/s12909-017-0967-3

Kotzer, A.M., Zacharakis, S.K., Raynolds, M., y Buenning, F. (2011). Evaluation of the built environment: staff and family satisfaction pre-and post-occupancy of the Children's Hospital. HERD: Health Environments Research y Design Journal, 4(4), 60-78. DOI: $10.1177 / 193758671100400405$

López-García, C., Ruiz-Hernández, J.A., Llor-Zaragoza, L., Llor-Zaragoza, P., y Jiménez-Barbero, J.A. (2018). User Violence and Psychological Well-being in Primary Health-Care Professionals. European Journal of Psychology Applied to Legal Context, 10(2), 57-63. DOI: 10.5093/ejpalc2018a6

Lown, B.A., McIntosh, S., Gaines, M.E., McGuinn, K., y Hatem, D. S. (2016). Integrating compassionate, collaborative care (the "Triple C") into health professional education to advance the triple aim of health care. Academic Medicine, 91(3), 310-316. DOI: 10.1097/ACM.0000000000001077

Luiz, F.F., Caregnato, R.C.A., y Costa, M.R.D. (2017). Humanization in the Intensive Care: perception of family and healthcare professionals. Revista Brasileira de Enfermagem, 70(5), 1040-1047. DOI: 10.1590/0034-7167-2016-0281

Mani, I., y Weese, J.S. (2016). Pet Therapy: Enhancing Patient Care Through Time with Animals. American Family Physician, 94(9), 737.

Martos, Á., Pérez-Fuentes, M.C, Molero, M.M., Gázquez, J.J., Simón, M.M, y Barragán, A.B. (2018). Burnout y engagement en estudiantes de Ciencias de la Salud. European Journal of Investigation in Health, Psychology and Education, 8(1), 23-36. DOI: 10.30552/ ejihpe.v8i1.223

Medeiros, A.C.D., Siqueira, H.C.H.D., Zamberlan, C., Cecagno, D., Nunes, S.D. S., y Thurow, M.R.B. (2016). Comprehensiveness and humanization of nursing care management in the Intensive Care Unit. Revista da Escola de Enfermagem da USP, 50(5), 816-822. DOI: 10.1590/s0080-623420160000600015

Miao, C., Humphrey, R.H., y Qian, S. (2017). A meta-analysis of emotional intelligence and work attitudes. Journal of Occupational and Organizational Psychology, 90(2), 177-202. DOI: 10.1111/joop. 12167

Molero, M.M., Pérez-Fuentes, M.C., Gázquez, J.J., y Barragán, A.B. (2018a). Burnout in health professionals according to their self-esteem, social support and empathy profile. Frontiers in Psychology, 9, 424. DOI: 10.3389/fpsyg.2018.00424

Molero, M.M, Pérez-Fuentes, M.C., Gázquez, J.J., Simón, M.M., y Martos, Á. (2018b). Burnout risk and protection factors in certified nursing aides. International Journal of Environmental Research And Public Health, 15(6), 1116. doi.org/10.3390/ijerph15 061116

Monti, F., Agostini, F., Dellabartola, S., Neri, E., Bozicevic, L., y Pocecco, M. (2012). Pictorial intervention in a pediatric hospital environment: effects on parental affective perception 
of the unit. Journal of Environmental Psychology, 32(3), 216-224. DOI: 10.1016/j.jenvp. 2012.03.001

Moretti, F., De Ronchi, D., Bernabei, V., Marchetti, L., Ferrari, B., Forlani, C., ... Atti, A.R. (2011). Pet therapy in elderly patients with mental illness. Psychogeriatrics, 11(2), 125-129. DOI: 10.1111/j.1479-8301.2010.00329.x

Organización Internacional de Normalización y Comisión Electrotécnica Internacional (2019). Guide to the development and inclusion of aspects of safety in International Standards for medical devices $\left(3^{a}\right.$ ed.). Recuperado de: https://www.iso.org/iso-guides.html

Oro, P., Esquerda, M., Viñas-Salas, J., Soler-González, J., y Pifarré, J. (2015). Mindfulness en estudiantes de medicina. FEM: Revista de la Fundación Educación Médica, 18(5), 305312.

Pérez-Fuentes, M.C., Molero, M.M., y Gázquez, J.J. (2019a). Explanatory Value of General SelfEfficacy, Empathy and Emotional Intelligence in Overall Self-Esteem of Healthcare Professionals. Social Work in Public Health, 34(4), 318-329. DOI: $10.1080 / 19371918.2019 .1606752$

Pérez-Fuentes, M.C., Molero, M.M, Gázquez, J.J, y Oropesa, N.F. (2018). The role of emotional intelligence in engagement in nurses. International Journal of Environmental Research and Public Health, 15(9), 1915. DOI: 10.3390/ijerph15091915

Pérez-Fuentes, M.C., Herrera-Peco, I., Molero, M.M., Oropesa, N.F., Ayuso-Murillo, D., y Gázquez, J.J. (2019b). The Development and Validation of the Healthcare Professional Humanization Scale (HUMAS) for Nursing. International Journal of Environmental Research and Public Health, 16, 3999. DOI:10.3390/ijerph16203999

Reeve, J., Lynch, T., Lloyd-Williams, M., y Payne, S. (2012). From personal challenge to technical fix: the risks of depersonalised care. Health y Social Care in the Community, 20(2), 145154. DOI: $10.1111 / \mathrm{j} .1365-2524.2011 .01026 . x$

Roohafza, H., Pirnia, A., Sadeghi, M., Toghianifar, N., Talaei, M., y Ashrafi, M. (2009). Impact of nurses clothing on anxiety of hospitalised children. Journal of Clinical Nursing, 18(13), 1953-1959. DOI: 10.1111/j.1365-2702.2008.02745.x

Segerstrom, S.C., Carver, C.S., y Scheier, M.F. (2017). Optimism. En M.D. Robinson y M. Eid (Eds.), The happy mind: Cognitive contributions to well-being (pp. 195-212). Cham, Switzerland: Springer International Publishing. DOI: 10.1007/978-3-319-58763-9_11

Seligman, M.E. (2006). Learned optimism: How to change your mind and your life. New York: Vintage Books.

Seligman, M.E., Schulman, P., y Tryon, A.M. (2007). Group prevention of depression and anxiety symptoms. Behaviour Research and Therapy, 45(6), 1111-1126. DOI: 10.1016/j.brat.2006.09.010

Shin, S., y Kang, J. (2019). Development and Validation of a Person-Centered Perioperative Nursing Scale. Asian Nursing Research, 13(3), 221-227. DOI: 10.1016/j.anr.2019.07.002

Tripodi, M., Siano, M.A., Mandato, C., De Anseris, A.G.E., Quitadamo, P., Nuzio, S.G., ... y Vajro, P. (2019). Humanization interventions in general pediatric wards: a systematic review. European Journal of Pediatrics, 178(5), 607-622. DOI:10.1007/s0043-01903370-3

Velasco, J.M., Heras, G. Ortega, A., y Gómez, V. (2017). Manual de buenas prácticas de humanización en Unidades de Cuidados Intensivos. Madrid: Proyecto HU-CI. Recuperado de: http://humanizandoloscuidadosintensivos.com/wpcontent/uploads/2017/11/Manual-Buenas Practicas-HUCI.pdf 
Vizoso, C.M., y Arias, O. (2018). Resiliencia, optimismo y burnout académico en estudiantes universitarios. European Journal of Education and Psychology, 11(1), 47-59. DOI: https://doi.org/10.30552/ejep.v11i1.185

Wersebe, H., Lieb, R., Meyer, A. H., Hofer, P., y Gloster, A. T. (2018). The link between stress, well-being, and psychological flexibility during an Acceptance and Commitment Therapy self-help intervention. International Journal of Clinical and Health Psychology, 18(1), 60-68. DOI: 10.1016/j.ijchp.2017.09.002

Recibido: 14 de abril de 2019

Recepción Modificaciones: 27 de abril de 2019

Aceptado: 8 de mayo de 2019 